\title{
EDITORIAL
}

Korean J Intern Med 2022;37:281-282

https://doi.org/10.3904/kjim.2022.065

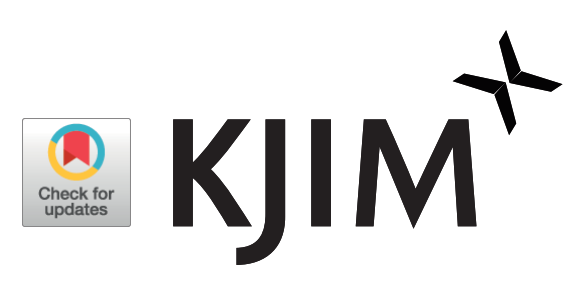

\section{A non-invasive test that enables longitudinal monitoring of chronic liver disease: VCTE versus 2D-SWE}

\author{
Hyunwoo Oh
}

Division of Gastroenterology, Department of Internal Medicine, Uijeongbu Eulji Medical Center, Eulji University, Uijeongbu, Korea

Received : February 16, 2022

Accepted: February 20, 2022

\section{Correspondence to} Hyunwoo Oh, M.D.

Division of Gastroenterology,

Department of Internal Medicine, Uijeongbu Eulji Medical Center, Eulji University, 712 Dongil-ro, Uijeongbu 11759, Korea

Tel: +82-31-951-1715,

Fax: +82-31-951-3300

E-mail: asklepios1258@eulji.ac.kr https://orcid.org/0000-0003-42248808
See Article on Page 285-293

Accurate evaluation of the severity of liver fibrosis and reliable diagnosis of cirrhosis are important steps for managing chronic liver disease (CLD), because they provide prognostic information and facilitate treatment decision-making [1]. The gold standard for diagnosis of liver fibrosis and cirrhosis is liver biopsy, but there is a risk of sampling errors and complications, and repeated testing to monitor clinical changes is not possible [2]. Against this background, noninvasive modalities for assessing and monitoring liver fibrosis are increasingly replacing liver biopsy. However, the weak correlation between modalities is problematic, and none has been identified as the gold standard [3].

The most intensively studied noninvasive method for assessing liver fibrosis is ultrasound-based shear wave elastography, including vibration-controlled transient elastography (VCTE) and two-dimensional shear wave elastography (2D-SWE). VCTE is simple and can be performed by unlicensed medical personnel. Therefore, it is used in many primary medical institutions and health centers. However, because the examination is performed without checking ultrasound images, accuracy is inadequate, and measurement is impossible in various clinical conditions such as severe obesity and ascites. Because 2D-SWE is a quantitative elastography method based on acoustic radiation force impulse and ultrasound images, positional accuracy is easier to achieve than with VCTE, even in patients with ascites or severe obesity. In addition, 2D-SWE allows operators to select a region of interest in the left lobe of the liver. However, 2D-SWE requires more technical expertise (and is thus typically conducted by a physician) and is time-consuming $[4,5]$.

In this issue of The Korean Journal of Internal Medicine, Yoo et al. [6] report a prospective cohort study involving 116 patients with CLD, in which they evaluated 2D-SWE (RS85 ultrasound system, Samsung Medison, Hongcheon, Korea) and VCTE (FibroScan, Echosens, Paris, France) for the assessment of liver fibrosis based on liver biopsy. The liver fibrosis stages were relatively evenly distributed (F0 [18\%], F1 [19\%], F2 [24\%], F3 [22\%], and F4 [17\%]). For the diagnosis of significant fibrosis ( $\geq$ F2), the area under the receiver operating-characteristic curve (AUROC) of 2D-SWE was 0.851, which was comparable to VCTE (0.859) and indicated good diagnostic ability, similar to a large-scale meta-analysis [7]. In this study, 2D-SWE was excellent for distinguishing advanced fibrosis ( $\geq F 3$ ), and VCTE for detecting cirrhosis ( $\geq$ F4) 
(AUROCs $=0.917$ and 0.938, respectively). The optimal cut-off values for predicting $\geq$ F2, F3, F4 of 2D-SWE were $5.83,7.55$, and $9.58 \mathrm{kPa}$, respectively. The proposed cutoffs require independent validation and vary slightly among studies $[7,8]$. The cut-off values should be tailored to each individual manufacturer and model. As reported previously, 2D-SWE had excellent inter-observer repeatability $[9,10]$. There was no difference in the relationship between stiffness (as measured by the two modalities) and liver histology according to the etiology of CLD.

The study excluded patients with liver disease and an aspartate aminotransferase or alanine aminotransferase level $>200 \mathrm{IU} / \mathrm{L}[6,8]$. According to a meta-analysis of the data of individual patients, this exclusion criterion has not been widely applied in previous studies. Although yet to be confirmed, the effects of alcohol and toxic hepatitis, which are difficult to evaluate by history-taking alone, and of fatty liver components in patients with chronic hepatitis $\mathrm{B}(\mathrm{CHB})$ and chronic hepatitis $C$, could be reduced. If data by liver disease etiology are given like the author's previous research [8], it is expected to be cited in more papers in the future.

Longitudinal monitoring of liver ultrasound in patients with CLD every 6 to 12 months will increase the usability of 2D-SWE, particularly for certain etiologies such as CHB [7]. Few clinics possess both VCTE and 2D-SWE instruments. Therefore, Yoo et al. [6]'s finding that one test alone is sufficiently reliable for diagnosing liver fibrosis based on liver biopsy is valuable, and demonstrates the clinical utility of 2D-SWE with RS85.

\section{Conflict of interest}

No potential conflict of interest relevant to this article was reported.

\section{REFERENCES}

1. Korean Association for the Study of the Liver (KASL). KASL clinical practice guidelines for liver cirrhosis: ascites and related complications. Clin Mol Hepatol 2018;24:230-277.

2. Bedossa P, Dargere D, Paradis V. Sampling variability of liver fibrosis in chronic hepatitis C. Hepatology 2003;38:1449-1457.

3. European Association for Study of Liver; Asociacion Latinoamericana para el Estudio del Higado. EASL-ALEH clinical practice guidelines: non-invasive tests for evaluation of liver disease severity and prognosis. J Hepatol 2015;63:237-264.

4. Kennedy P, Wagner M, Castera L, et al. Quantitative elastography methods in liver disease: current evidence and future directions. Radiology 2018;286:738-763.

5. Dietrich CF, Bamber J, Berzigotti A, et al. EFSUMB guidelines and recommendations on the clinical use of liver ultrasound elastography, update 2017 (long version). Ultraschall Med 2017;38:e16-e47.

6. Yoo HW, Kim SG, Jang JY, et al. Two-dimensional shear wave elastography for assessing liver fibrosis in patients with chronic liver disease: a prospective cohort study. Korean J Intern Med 2022;37:285-293.

7. Herrmann E, de Ledinghen V, Cassinotto C, et al. Assessment of biopsy-proven liver fibrosis by two-dimensional shear wave elastography: an individual patient data-based meta-analysis. Hepatology 2018;67:260-272.

8. Yoo JJ, Kim SG, Kim YS. The diagnostic accuracy of LOGIQ S8 and E9 shear wave elastography for staging hepatic fibrosis, in comparison with transient elastography. Diagnostics (Basel) 2021;11:1817.

9. Kaposi PN, Unger Z, Fejer B, et al. Interobserver agreement and diagnostic accuracy of shearwave elastography for the staging of hepatitis C virus-associated liver fibrosis. J Clin Ultrasound 2020;48:67-74.

10. Yoo J, Lee JM, Joo I, Yoon JH. Assessment of liver fibrosis using 2-dimensional shear wave elastography: a prospective study of intra- and inter-observer repeatability and comparison with point shear wave elastography. Ultrasonography 2020;39:52-59. 